\title{
A System for Automatic Judgment of Offsides in Soccer Games
}

\author{
Sadatsugu Hashimoto \\ Shinji Ozawa \\ Department of Information and Computer Science, Keio University \\ 3-14-1 Hiyoshi, Kouhoku-ku, Yokohama 223-8522, Japan \\ \{bridge_book, ozawa\}@ozawa.ics.keio.ac.jp
}

\begin{abstract}
In this paper, we propose a system for automatic judgment of offsides in soccer games. We detect and track players in fixed multi camera images and calculate the world coordinates of them. Furthermore, we do a formation analysis by classifying uniforms and calculate the position of an offside line. On the other hand, we calculate the $3 D$ coordinates and the trajectories of a ball in world coordinates from the plane coordinates of a ball in multi cameras and recognize the moment of a play from the $3 D$ trajectories of a ball. In addition, we make a judge player's interfering with play by analyzing the spatial relationship between a ball and players. Finally, we make an offside judgment by integrating these results. We apply our system to a real soccer match and demonstrate the availability of this system by showing the experimental results.
\end{abstract}

\section{INTRODUCTION}

Recently, various researches have done on sports scene analysis. For example, detection and tracking of players and ball in sports video, especially soccer, are done [1][2], and these results are applied to entertainment applications such as 3D scene reconstruction [3] [4] and broadcasting technology such as intelligent robot camera in TV programming production, but not applied to judgments of games. As a few example of applying image processing to judgment of game, there is a judgment of games in American football. Objection by coaches being in a judgment coal, even if important scenes are not taken from the most suitable direction, by creating the angles with technology of $\mathrm{CG}$, precise judgments is made. However, after all, referees who watch the movie make judgments, not automatically.

So we make judgments of games in real sports matches automatically with image processing. Especially, we pay our attention to soccer that is one of most popular sports in the world and expose a focus to an offside judgment, since it is the most difficult judgment in soccer games and often brings controversies. As for the reason, in order to make an offside judgment, recognizing the positions and team information of all players precisely and deciding the position of an offside line, recognizing the moment of passes, and making judgment player's interfering with play is required. Doing these works by one linesman in a very wide soccer field at once is very difficult, but image processing enables to do them. So, in this paper, we propose a system for automatic judgment of offsides in soccer games with image processing. By using multiple view images taken by fixed cameras, we calculate the position of an offside line by tracking all players precisely and team classification, furthermore, recognize the moment of passes from the 3D trajectory of a ball. In addition, we make a judge player's interfering with play by analyzing the spatial relationships between a ball and players. Finally, we make offside judgments automatically by integrating these results.

\section{SYSTEM OVERVIEW}

Here, the rule of offside in soccer is mentioned simply. The position of the player who is second nearest to the goal among a defensive team is the position of an offside line. The player who is nearer to the goal than the offside line is to be in an offside position. If the player who was in an offside position at the moment of the play of a pass forward by an offensive player interfere next play, offside judgment is made.

Therefore, this system is composed of roughly three stages. One is offside line calculation based on player tracking and team classification. Another is play recognition by $3 \mathrm{D}$ trajectories of a ball and the spatial relationships between a ball and players. The last one is making offside judgments by integrating these results.

In this system, in order to track players robustly to occlusion and calculate the 3D coordinates of a ball precisely, 16 fixed cameras are used, which are located along the both longest sides of the soccer field to cover all the area and are in parallel with a soccer field like Fig.1. 


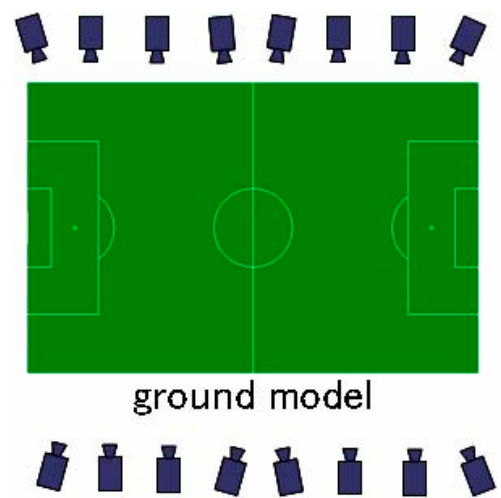

Fig.1 System Environment

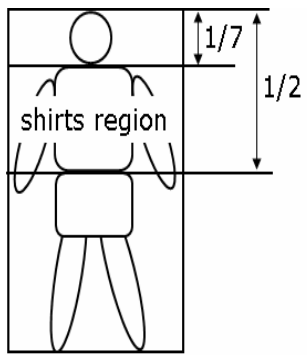

Fig.2 Human Model

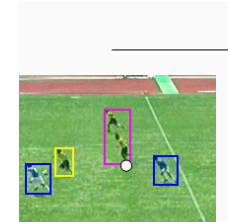

occlusion
Sorted camera images

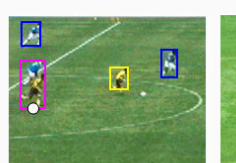

occlusion

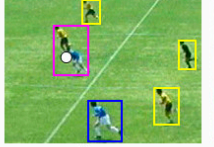

occlusion

$\bigcirc$ : projected point

$\square$ : one team player region

: the other team player region

$\square$ : occluded player region

Fig.3 Player Tracking

\section{OFFSIDE LINE CALCLATION}

In order to calculate the position of an offside line, it is necessary to obtain the world coordinates and team information of all players and do a formation analysis based on these features. First, we introduce the method of player detection and tracking, second, we describe how to do a formation analysis.

\subsection{Player Detection and Tracking}

First, in inner-camera operation, we calculate player's foot coordinates, make occlusion judgments and do uniform classification independently in each camera. Next, in intercamera operation, those features of 16 cameras are integrated for player tracking.

\subsubsection{Inner-camera operation}

First, as pre-processing, background subtraction is done on input images to extract player regions, and binary images is made by using thresholds of intensity and area with noise removal and connection processing. Then each region of player is labeled and extracted. Second, each detected player region is classified into a non-occluded player region or an occluded one. In our method, occlusion judgment is made based on the threshold of the height and area of player regions since the occluded player regions must be large. But the height and area of each player changes in according to the position on images, that is, the distance from cameras. So, we make threshold level formality by y coordinate in plane coordinates. For each detected non-occluded player region, its center, $(\mathrm{x}, \mathrm{y})$ coordinates of which the $\mathrm{y}$-value is the largest, which is equal to the foot coordinates is calculated as a player feature. Third, we classify nonoccluded players regions into 5 uniforms with two-team field player, goal keeper and referee. Comparing the distance between the average value of color statistics $(R$, $\mathrm{G}, \mathrm{B}, \mathrm{H}, \mathrm{S}, \mathrm{L}$ ) of each player region with that of each 5 uniform registered before the game, the uniform color of the nearest distance is the one of the player. The mahalanobis distance is used for measuring color distance and color value acquisition region is only a shirt region based on human model like Fig.2.

\subsubsection{Inter-camera operation}

In our method, each player is tracked independently. Based on the world coordinates of the tracking player in pre-frame, 4 optimum cameras are sorted and chosen among 16 cameras for tracking. Sorting and choosing camera is based on the area which the tracking player is in and the player is to be nearer to the cameras. As the player is nearer to the camera, the player region is bigger in input images and the accuracy of background subtraction and calculation of foot coordinate is higher. The world coordinates of the tracking player in pre-frame is projected by a homography on chosen camera images in sorted order. Among the projected point, if there exists the player region which is non-occluded and has same uniform color of the tracking player, the player region is tracking player region. If there exists the region a least one camera, tracking is possible like Fig.3. Abovementioned operation is processed for all players. Thus, by using multi-view-camera, we can track players more robustly to occlusion than the method that use single camera.

\subsection{Formation Analysis}

The number of detected and tracked regions is 25 including two team players and three referees. Based on the world coordinates(x) and uniform color information of these regions, we do a formation analysis. We detect the second nearest player to the goal of one team and determine this player's x-coordinate as the position of offside line. Comparing the position of the offside line and the players of the other one, if there exists some player nearer to the goal than the offside line, the player is to be in an offside position. 


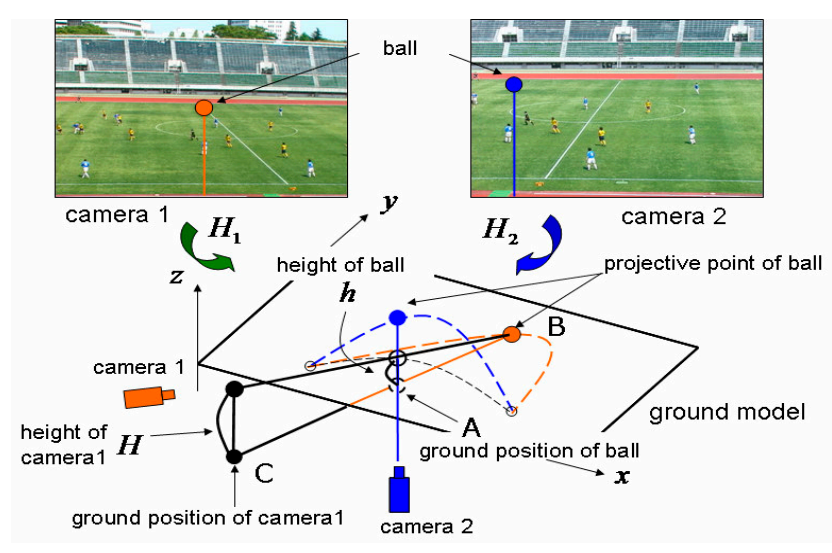

Fig.4 3D Coordinates Calculation of Ball

\section{TARGET PLAY FOR OFFSIDE JUDGMENT DETECTION}

First, we calculate the 3D coordinates of a ball in world coordinates from the plane coordinates of a ball in two adjacent cameras with the geometry relation between input images and ground model. Calculating the 3D coordinates of a ball every frame leads the 3D trajectory of a ball. Second, we detect the moment of plays from the change of the trajectory of a ball. Third, we recognize the target play of an offside judgment by analyzing the spatial relationships of a ball and players at the moment of the play and ball's vectors. In this paper, in order to obtain the $3 \mathrm{D}$ coordinates of a ball precisely, detecting and tracking a ball in input images is done almost manually, but automatic tracking methods such as [5][6][7] also can be applied.

\subsection{D Coordinates Calculation of Ball}

We use two plane coordinates of a ball in adjacent cameras to calculate 3D coordinates of ball. First, we calculate the ground coordinates(x,y) of a ball. Each camera being located in parallel with the field, the point of intersection with the line which took down a perpendicular line from a ball and the field is a ground position of a ball in an input image like Fig.4. Since there should be the ground coordinates of a ball in world coordinates on the line which is projected the perpendicular line on the ground model. So we project two perpendicular lines from two input images on the ground model and assume the point of intersection of the two projected lines as the ground coordinates of a ball in world coordinates. Second, we apply Kim's method [8] to calculate the height (z-coordinate) of a ball. From the triangular geometric relation shown in Fig.4, the height of a ball can be calculated. Thus, 3D coordinates of a ball in world coordinates can be calculated. Doing this process every frame leads the 3D trajectory of a ball like small circles in Fig.5.

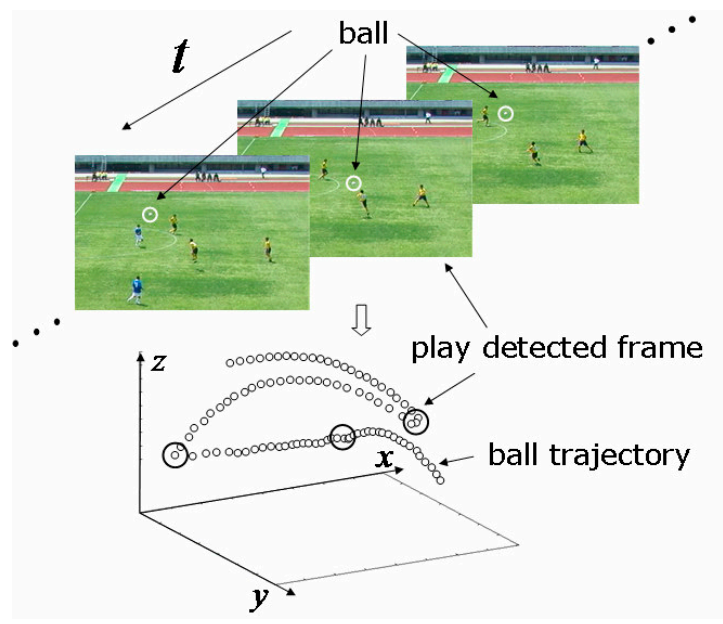

Fig.5 Play Detection

\subsection{Play Detection}

We detect the moment when some play occurs by the trajectory of a ball. As for the moment when some play occurs, the trajectory of a ball changes, that is to say, the acceleration of a ball must be large. So we calculate the acceleration of a ball from the world coordinates of a ball and detect the moment when acceleration is big as the moment when some play occurs. The example of the moment when some play occurs is shown as big circle in Fig. 5.

\subsection{Play Judgment}

We examine the region around a ball at the moment when play occurs, the nearest player to a ball is regarded as the player who interfere with play. If there exists no player near the ball, the change of trajectory of a ball is result from ball's bound. The nearest player to a ball being an offense player and the vector of ball being forward at the moment when play occurs, the play is the pass forward by offensive player and the target play of offside judgments.

\section{OFFSIDE JUDGMENT}

In order to do an offside judgment, it is necessary to judge whether the player who was in an offside position at the moment of the play of a pass forward by offensive player interferes with a next play or not. So we do an offside judgment as follows

\section{Step.1}

The player who is in an offside position at the moment of the play of a pass forward by offense player is to be a target player of offside judgment. 

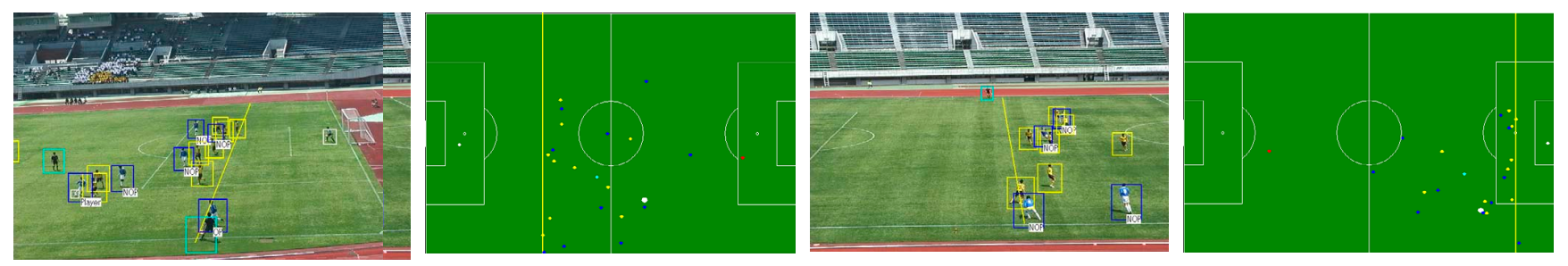

Fig.6 The result of Offside Line Calculation and Offside Position Judgment (Left: Non-offside scene; Right: Offside-scene)

\section{Step.2}

When the next play is detected, if the player who was in an offside position is in the position where is the nearest to ball, it is regarded that he interferes with play and we make offside judgments.

\section{EXPERIMENTAL RESULT}

We apply our system to one real soccer match. Input image sequences are digitized of $720 \times 480$ pixels, RGB 24 bit and 15 frame/sec. The second and forth image in Fig.6 present the example results of formation analysis and offside line calculation on ground model at the moment when the play of a pass forward by offensive player is detected. The first and third image present the result of offside line calculation by projecting the world coordinates (x-coordinate) of an offside line on an input image by using a homography and offside position judgment by displaying offside-position players as "OP" and non-offside-position players as "NOP" at the moment. Here, we can see that an offside line is under the foot position of second nearest defensive player to the goal including keeper and offensive players is judged whether being in an offside position or not precisely. Next, we describe the result of offside judgments. In the match which we apply this system to, offside scene is two including the right scene in Fig.6. This system made offside judgments toward both scenes. In the two scenes which referees made offside judgments by mistake in spite of not offside scene including the left scene in Fig.6, this system made non-offside judgments precisely.

\section{CONCLUSION}

We have proposed a system for automatic judgment of offsides in soccer games. Using multi view cameras enables robust player tracking and calculating the 3D coordinates of a ball precisely. Robust player tracking enables to calculate the position of an offside line precisely and calculating 3D ball trajectory enables to detect the moment of play. In addition, player's interfering with play can be recognized by analyzing spatial relationship of a ball and players.
Finally, offside judgment can be made automatically by integrating these results. Now, we experience on only one soccer match, but we apply our system to more soccer matches and scenes in future work. Furthermore, other offside rules which we don't target in this paper, like that the player who is in an offside position interferes with an opponent team player and the player gains advantage by being in offside position, is required to considered in future work.

\section{REFERENCES}

[1] T.Misu, M.Naemura, W.Zheng, Y.Izumi, and K.Fukui, "Robust tracking of soccer players based on data fusion," Proc. ICPR 2002, vol.1, pp. 556-561, Aug.2002.

[2] Xu.M, Orwell.j, Lowey.L, Thirde.D, "Architecture and algorithms for tracking football players with multiple cameras," Vision, Image and Signal Processing, IEE Proceedings, vol.152, Issue.2, pp. 232-241, April.2005.

[3] Y.Kameda, T.Koyama, Y.Mukaigawa, F.Yoshikawa and Y.Ohta, "Free Viewpoint Browsing of Live Soccer Games," Proc. ICME 2004, pp. 747-750, June. 2004.

[4] Inamoto.N, Saito.H, "Free Viewpoimt Video Synthesis and Presentation from Multiple Sporting Videos," Proc. ICME 2005, pp. 326-329, July. 2005.

[5] Xinguo.Yu, Qi.Tian, Kong Wah Wan, "A novel ball detection framework for real soccer video," Proc. ICME 2003, vol.2, pp. 265-268, July 2003.

[6] Xiao-Feng Tong, Han-Qing Lu, Qing-Shan Liu, "An effective and fast soccer ball detection and tracking method," Proc. ICPR 2004, vol. 4, pp. 795-798, Aug. 2004.

[7] Xinguo $\mathrm{Yu}$, Changshen $\mathrm{Xu}$, Qi Tian, Hon Wai Leong, "A ball tracking framework for broadcast soccer video," Proc. ICME 2003, vol. 2, pp. 273-276, July. 2003.

[8] T.Kim, Y.Seo, K.sang, "Physics-Based 3D Position Analysis of a Soccer Ball from Monocular Image Sequences," Proc. ICCV 1998, pp. 721-726, 1998. 